\title{
Herz und Gehirn - eine klinisch bedeutsame Beziehung
}

Lüscher, Thomas F ; Roffi, Marco

DOI: https://doi.org/10.1007/s00059-008-3178-1

Other titles: The Heart and Brain - a clinically significant situation relationship

Posted at the Zurich Open Repository and Archive, University of Zurich ZORA URL: https://doi.org/10.5167/uzh-156188

Journal Article

Published Version

Originally published at:

Lüscher, Thomas F; Roffi, Marco (2008). Herz und Gehirn - eine klinisch bedeutsame Beziehung. Herz, 33(7):473-474.

DOI: https://doi.org/10.1007/s00059-008-3178-1 


\section{Herz und Gehirn - eine klinisch bedeutsame Beziehung}

\author{
Thomas F. Lüscher ${ }^{1}$, Marco Roffi²
}

Herz und Hirn sind zentrale Organe des menschlichen Körpers. Das Herz bleibt auch nach seiner Entweihung als Symbol und Hort unserer Gefühle im Mittelpunkt unseres Denkens und Fühlens, während das Hirn als Sitz unseres Selbst Bedeutung erlangt. In der Medizin werden die beiden Organe von unterschiedlichen Spezialisten betreut: Kardiologen befassen sich mit dem Herz und Neurologen mit dem Hirn, und dies, obwohl beide Organe eigentlich eng zusammengehören (Abbildung 1, Tabelle 1). Einerseits ermöglicht das Herz erst dem Hirn das Denken, andererseits haben viele Erkrankungen des Hirns ihre Ursache im Herzen. Die wichtigsten Erkrankungen beider Organe - Herzinfarkt und Hirnschlag - haben zudem häufig die gleiche Ursache: die Atherosklerose. Ein Karotisgeräusch bei der Auskultation oder eine Karotisplaque in der Ultraschalluntersuchung sind wichtige Hinweise auf das Hirnschlagrisiko und das Vorhandensein einer koronaren Herzkrankheit. Große Studien haben gezeigt, dass die Wanddicke der Halsschlagader, die sog. ,,intima-media-thickness“ (IMT), mit zukünftigen kardiovaskulären Ereignissen in enger Beziehung steht. In der vorliegenden Ausgabe von Herz gehen A. M. Shah, T. Banerjee und D. Mukherjeee, Lexington, USA, dieser komplexen Beziehung zwischen Gefäßveränderungen in den Koronargefäßen, den peripheren Arterien und zerebrovaskulären Gefäßen eingehend nach [1].

Die Darstellung der zerebrovaskulären Gefäße bereitete anfänglich große Schwierigkeiten. Seit der Einführung der Ultraschalluntersuchung, vor allem der Magnetresonanztomographie und der Computertomographie, gelingt es heute, die Gefäße, welche das Hirn versorgen, in ihrer Struktur mit beeindruckender Auflösung darzustellen (Abbildung 2). Demzufolge ist eine Katheterangiographie für diagnostische Zwecke selten nötig. M. Siebler aus Düsseldorf und J. B. Fiebach aus Berlin beschreiben umfassend die Bildgebung extra- und intrakranieller zerebrovaskulärer Gefäße sowie ihre diagnostische Bedeutung [2].

Die arterioarterielle Embolie bei Patienten mit Karotisstenose ist eine wichtige Ursache des ischämischen Hirnschlags. Die Atherosklerose befällt klassischerweise die Arteria carotis interna unmittelbar an ihrem Ursprung bei der Karotisgabel. Das Management von Patienten mit Karotisstenose ist heute viel facettenreicher geworden, seit neben der medikamentösen Therapie (vor allem mit Antithrombotika und Antihypertensiva sowie Lipidsenkern) und der chirurgischen Endarteriektomie nun auch das Karotisstenting verfügbar ist. T. Lüscher, Zürich, und M. Roffi, Genf, behandeln in einem umfassenden Beitrag das moderne Management der Karotisstenose und insbesondere den differentialtherapeutischen Einsatz der o.g. Behandlungsmöglichkeiten [3].

Auch die Aorta kann als Quelle von Embolien in die zerebrovaskuläre Zirkulation eine wichtige Rolle beim Auftreten von transienten ischämischen Attacken und beim Hirnschlag spielen. Ähnliches gilt für Veränderungen der Aortenklappe sowie des Herzens selbst. Embolien aus thrombotischen Auflagerungen im Bereich eines großen Herzinfarkts (vor allem bei Verschluss des Ramus interventricularis anterior mit Vorderwandinfarkt) sind seit Einführung der akuten perkutanen Koronarintervention zwar seltener geworden; da die Infarkte heute deutlich kleiner sind und damit auch die Gefahr einer Thrombusbildung im linken Ventrikel geringer geworden ist, müssen sie aber

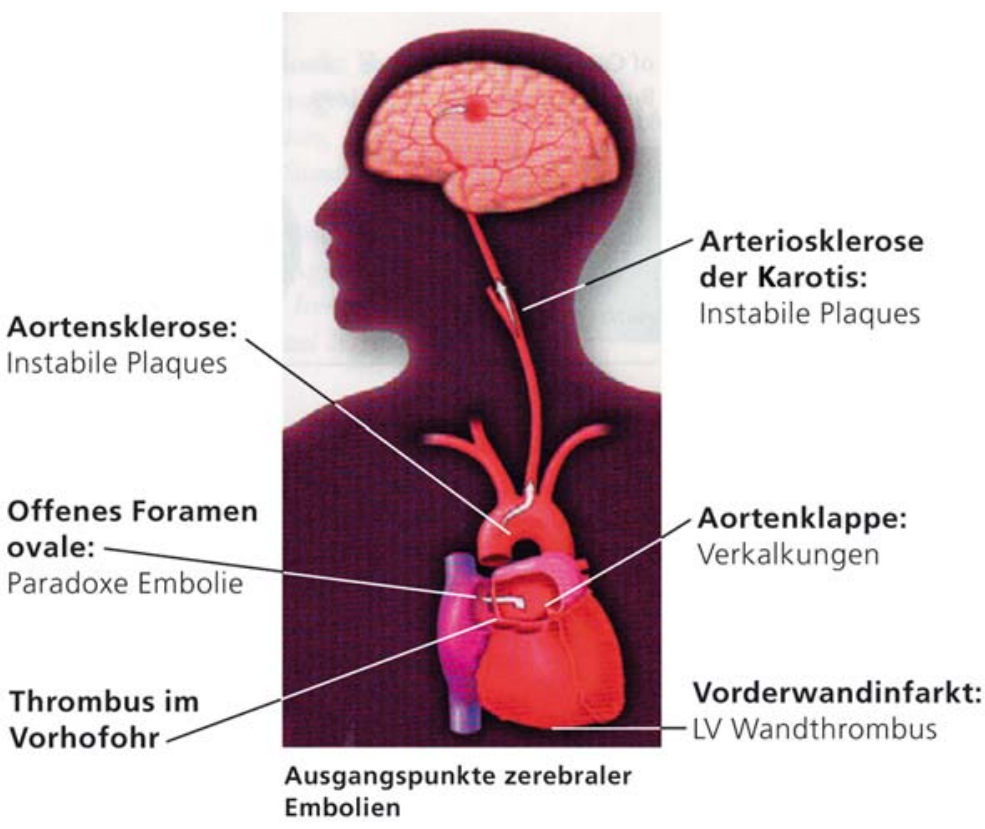

Abbildung 1. Das Herz als Ursache embolischer Ereignisse in der Zerebralzirkulation (Abdruck mit Genehmigung von D. Hermann).

Figure 1. The heart as a source of embolic events in the cerebrovascular circulation (reprint by courtesy of D. Hermann).

\author{
Herz-Kreislauf- \\ Zentrum, Klinik \\ für Kardiologie, \\ Universitätsspital \\ Zürich, Schweiz \\ 2 Service de Cardiologie, \\ Département de \\ Médecine Interne, \\ Hôpitaux Universi- \\ taires de Genève, \\ Schweiz.
}

Herz 2008;33:473-4

DOI 10.1007/

s00059-008-3178-1 


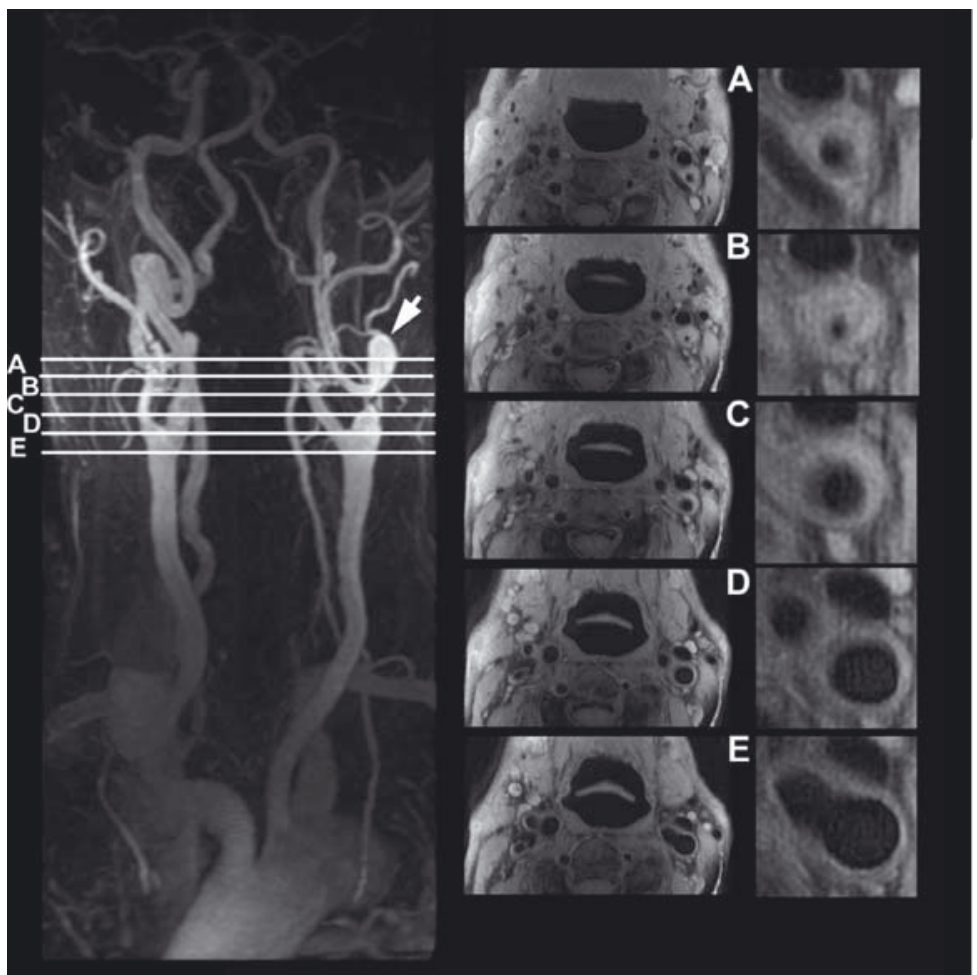

Abbildung 2. Magnetresonanztomographie der Arteria carotis mit Einengung der Arteria carotis interna (Abdruck mit Genehmigung von R. Corti).

Figure 2. Magnetic resonance imaging of the carotid artery with narrowing of the internal carotid artery (reprint by courtesy of R. Corti).

Tabelle 1. Beziehung zwischen Herz und Hirn bei kardiovaskulären Erkrankungen und Risikofaktoren.

Table 1. Relation between heart and brain cardiovascular diseases and risk factors.

\begin{tabular}{|c|c|}
\hline Herz & Hirn \\
\hline Hypertonie & Hirnblutung, ischämischer Insult \\
\hline \multirow[t]{2}{*}{ Hyperlipidämie } & Karotis- und Aortenplaques mit Embolie \\
\hline & Paradoxe Embolie und Hirnschlag \\
\hline Offenes Foramen ovale & Embolischer Hirnschlag \\
\hline Vorhofflimmern, Vorhofthromben & Embolischer Hirnschlag \\
\hline Vorderwandinfarkt mit Wandthrombus & Synkope, embolischer Hirnschlag \\
\hline Aortenstenose & $\begin{array}{l}\text { Embolischer Hirnschlag, Synkope, } \\
\text { Schwindel }\end{array}$ \\
\hline Aortenplaques & Embolischer Hirnschlag \\
\hline $\begin{array}{l}\text { Herzinsuffizienz mit linksventrikulärer } \\
\text { Dysfunktion (Auswurffraktion < 30\%) }\end{array}$ & $\begin{array}{l}\text { Hypoperfusion mit Funktionsstörungen, } \\
\text { Apathie, Depression, Synkope, Schwindel }\end{array}$ \\
\hline
\end{tabular}

dennoch differentialdiagnostisch in Erwägung gezogen werden.

Thromben im Vorhof finden sich vor allem bei Vorhofflimmern (Abbildung 1), einer der häufigsten Rhythmusstörungen überhaupt; gerade hier kommt der medikamentösen Therapie mit Gerinnungshem- mern und Angiotensin-Rezeptor-Antagonisten sowie in Zukunft der Ablationstherapie eine enorme Bedeutung zu. In den letzten Jahren wurde eine paradoxe Embolie durch ein offenes Foramen ovale ebenfalls als Quelle von Embolien in die zerebrovaskuläre Zirkulation erkannt. Neben der Blutverdünnung wird in dieser Patientengruppe zunehmend auch der Verschluss eines offenen Foramen ovale mit einem perkutan eingeführten Verschlussdevice als Therapieoption angeboten.

Das Management des akuten Hirnschlags ist immer noch schwierig und komplex, obschon sich in den letzten Jahren neue Möglichkeiten etabliert haben. Dabei spielt vor allem die Fibrinolyse eine wichtige Rolle, welche kompetent von F. Orso, S. Baldasseroni und A. P. Maggioni aus Florenz, Italien, besprochen wird [4]. Neben der peripheren Fibrinolyse kommt vor allem in erfahrenen Zentren vermehrt die katheterbasierte Intervention bei Patienten mit akutem Stroke mit z.T. beeindruckenden Ergebnissen zum Zuge. Die Möglichkeiten und Grenzen dieser eleganten Interventionsmöglichkeit werden versiert von Gerhard Schroth aus Bern, Schweiz, in einem Übersichtsartikel besprochen [5].

Vorbeugen ist besser als heilen - dieser Leitsatz gilt beim Hirnschlag im Besonderen, da bei einem zerebrovaskulären Ereignis unwiederbringlich Neuronen untergehen, die nur teilweise durch die Plastizität des Hirns ersetzt werden können. Entsprechend kommt der Hirnschlagprävention besondere Bedeutung zu. J. Ederle, Heidelberg, und M. M. Brown, London, UK, besprechen umfassend die heutigen Möglichkeiten der Hirnschlagprävention und gehen insbesondere auf die Bedeutung der Blutdruckeinstellung, der Cholesterinsenkung und der Kontrolle anderer Risikofaktoren ein [6].

\section{Literatur}

1. Shah AM, Banerjee T, Mukherjee D. Coronary, peripheral and cerebrovascular disease: a complex relationship. Herz 2008;33:475-80.

2. Siebler $M$, Junghans $U$, Fiebach JB. Bildgebung bei zerebrovaskulärer Erkrankung. Herz 2008;33:482-8.

3. Roffi M. Lüscher TF. Management of patients with carotid artery stenosis. Herz 2008;33:490-7.

4. Orso F, Baldasseroni S, Maggioni AP. The role of thrombolysis in acute ischemic stroke. Herz 2008;33:498-506.

5. Gralla J, Brekenfeld C, Arnold M, Schroth G. Acute Stroke: Present and Future of catheter-based interventions. Herz 2008;33:507-17.

6. Ederle J, Brown MM. Stroke prevention. Herz 2008;33: 518-23.

\section{Korrespondenzanschrift}

Prof. Dr. Thomas F. Lüscher, FRCP, FESC

Direktor der Klinik für Kardiologie

Herz-Kreislauf-Zentrum

Universitätsspital

8091 Zürich

Schweiz

Telefon (+41/44) 255-2121, Fax -4251

E-Mail:karlue@usz.unizh.ch 